\title{
Pairwise antibiotic interactions in Escherichia coli: triclosan, rifampicin and aztreonam with nine other classes of antibiotics
}

\author{
Caroline Nguyen ${ }^{1}$, Alice Zhou ${ }^{1}$, Ayesha Khan ${ }^{1}$, Jeffrey H Miller ${ }^{1}$ and Pamela Yeh $^{2}$ \\ Previous investigations have shown that analyzing pairwise interactions between 22 antibiotics in Escherichia coli enable the \\ grouping of drugs into nine functional classes. Our aim is to significantly expand this drug network by adding three clinically \\ relevant antibiotics, two of which have distinct mechanisms of action not previously represented. Here, we examine triclosan, \\ rifampicin and aztreonam, by testing them pairwise against themselves and one antibiotic from each of the nine classes. \\ Network analysis shows that triclosan, rifampicin and aztreonam each form distinct functional groups of their own. This is \\ particularly surprising for aztreonam, which is a cell wall synthesis inhibitor but did not cluster with other cell wall synthesis \\ inhibitors. Furthermore, of the $\mathbf{3 0}$ pairs tested, we found several previously unreported synergies that could potentially be used in \\ multidrug therapies. The distinct functional groups suggest that there may be properties of these drugs that are different from \\ other drugs even with similar mechanism of action, and that, when considering drug combinations, aztreonam in particular is not \\ simply interchangeable with other cell wall synthesis inhibitors.
}

The Journal of Antibiotics (2016) 69, 791-797; doi:10.1038/ja.2016.26; published online 9 March 2016

\section{INTRODUCTION}

The proliferation of multidrug resistant pathogens is a major problem in public health., ${ }^{1,2}$ Gram-negative pathogens present additional challenges, as the outer membrane renders many antibiotics ineffective against these bacteria. ${ }^{3}$ Combinatorial strategies for antibiotic use offer an expanding repertoire of drug therapies (see reviews)., ${ }^{4,5}$ Previous studies have shown that pairwise combinations of antibiotics in Escherichia coli can be used to construct an interaction network, ${ }^{6}$ which can then be used to classify drugs into different groups based on the premise that each group will interact with each other group synergistically, antagonistically or additively. When clustered in such a way, the drugs end up in groups based on their mechanism of action. ${ }^{6}$

Here, our goal is to increase our understanding of drug networks by expanding the interaction network to include three medically important and commonly used antibiotics: rifampicin (RIF), triclosan (TRI) and aztreonam (AZM; see Figure 1). Rifampicin inhibits most prokaryotic RNA polymerases. ${ }^{7,8}$ It is used in combination with several drugs for different infections, including tuberculosis and leprosy. ${ }^{8}$ Rifampicin is also used in combination with fusidic acid (FUS) for a number of infections, such as MRSA (methicillin-resistant Staphylococcus aureus). ${ }^{9}$ It has been reported to be antagonistic with the aminoglycoside gentamicin (GEN). ${ }^{10}$ TRI is widely used in detergents, soaps and many other household products, as well as in surgical scrubs and hand washes. ${ }^{11,12}$ TRI inhibits fatty acid biosynthesis by binding to the FabI gene product, ${ }^{12,13}$ but at high concentrations may have additional targets, in particular the bacterial membrane. ${ }^{11}$ Although drug combinations with TRI may not be feasible clinically, being able to include TRI in the drug interaction network would be valuable, as we might be able to pinpoint the mechanism of newly discovered antimicrobials that fall into the same group based on pairwise tests. AZM is a monobactam that is resistant to some $\beta$-lactamases that cleave penicillins, ${ }^{14}$ and is used against Gram-negative infections. It has known synergy with aminoglycosides (for example, tobramycin). ${ }^{15}$ Here, we show that each of these antibiotics (RIF, TRI and AZM) form a unique functional group, based on an analysis of their pairwise interactions with representatives of each of the nine different functional classes of antibiotics defined in previous studies. ${ }^{6,16} \mathrm{We}$ also found several novel strong synergies.

\section{MATERIALS AND METHODS}

Antimicrobials

Antibiotics used include: AZM, RIF, ampicillin (AMP), chloramphenicol (CHL), ciprofloxacin (CPR), clindamycin (CLI), FUS, GEN, nitrofurantoin (NIT), trimethoprim (TMP) and vancomycin (VAN). Antimicrobial agent TRI was also used in the study. All were purchased from Sigma (St Louis, MO, USA). For use in our experiments, all solid antibiotics were first dissolved in water, ethanol or methanol to create stock solutions of $10 \mathrm{mg} \mathrm{ml}^{-1}$. Stocks were then stored in $200 \mu \mathrm{l}$ aliquots at $4^{\circ} \mathrm{C}$. For each experiment, one $200 \mu \mathrm{l}$

${ }^{1}$ Department of Microbiology, Immunology, and Molecular Genetics, the Molecular, Biology Institute, and the David Geffen School of Medicine, University of California, CA, USA and ${ }^{2}$ Department of Ecology and Evolutionary Biology, University of California, CA, USA

Correspondence: Dr P Yeh, Department of Ecology and Evolutionary Biology, University of California, Los Angeles, Los Angeles, CA 90095, USA.

E-mail: pamelayeh@ucla.edu

Received 19 October 2015; revised 31 December 2015; accepted 1 February 2016; published online 9 March 2016 
aliquot of each antibiotic stock was thawed and used (see Growth conditions section).

\section{Strains and media}

The E. coli strain used here for all experiments is the starting strain for the KEIO collection, ${ }^{17}$ strain BW25113. ${ }^{18}$ This strain is: $\operatorname{lac}^{q 1} \operatorname{rrnB}_{T 14} \Delta$ lac $Z_{W J 16}$ hsdR514 $\triangle a r a B A D_{A H 33} \Delta r h a B A D_{L D 78}$. The following medium was used: LB (10 $\mathrm{g}$ tryptone, $5 \mathrm{~g}$ yeast extract, $10 \mathrm{~g} \mathrm{NaCl}$ per liter). ${ }^{19}$

\section{Growth conditions}

Unless otherwise stated, all genetic methods and manipulations (for example, sterile technique, plating for single colonies and diluting) are as given in Miller. ${ }^{19}$ Over-day cultures were prepared daily by adding $20 \mu$ l of a $2 \times 10^{9}$ cells per $\mathrm{ml}$ magnesium chloride buffer cell stock to $2 \mathrm{ml}$ of LB. They were then grown for $6 \mathrm{~h}$ in a $37^{\circ} \mathrm{C}$ incubator. Experimental cultures containing different concentrations of antibiotics were then prepared by diluting the appropriate amount of antibiotic solution into $2 \mathrm{ml}$ of LB per culture to achieve the desired concentrations. Each culture was then seeded with $\sim 10^{3}$ cells by inoculating them with $50 \mu \mathrm{l}$ of a $10^{-4}$ dilution of the over-day culture. The cultures were then incubated on a rotor at $37^{\circ} \mathrm{C}$ and 50 r.p.m. for $18 \mathrm{~h}$ over-night. After

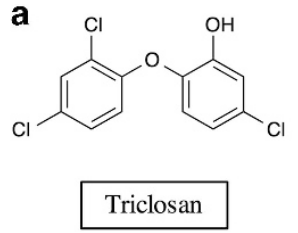

b
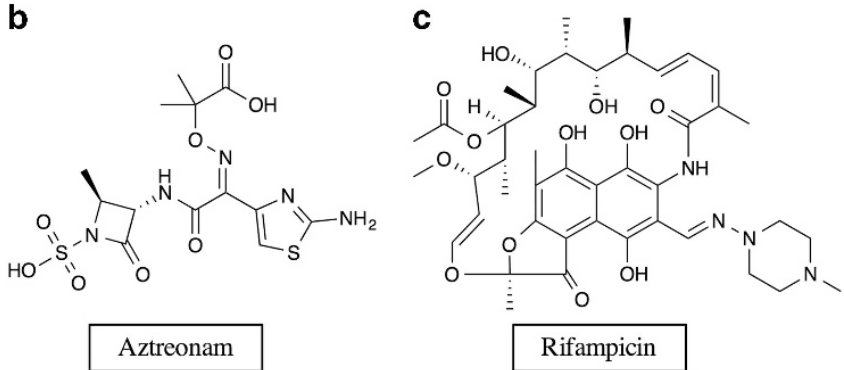

Figure 1 Molecular structures of triclosan, aztreonam and rifampicin. (a) Triclosan has a two ring aromatic structure. (b) Aztreonam is a bactericidal synthetic monocyclic beta-lactam. (c) Rifampicin is a bactericidal drug of the rifamycin family.
$18 \mathrm{~h}$, the $\mathrm{OD}_{600}$ of each culture was measured to ascertain cell growth. Graphs of these data display percent growth versus that in $\mathrm{LB}$ alone.

Determination of MICs and single-drug concentrations to be used in interaction experiments

To determine the MIC, we followed methodology provided by Andrews. ${ }^{20} \mathrm{We}$ used LB media. Overnight cultures containing a range of concentrations of a given antibiotic (usually serial dilutions from the reported MIC in twofold intervals) were prepared. To examine bacteria growth rates in single-drug and two-drug environments, we used sub-ICs that resulted in bacterial growth of 50-90\% compared with bacterial growth in no drug environments (Table 1). These concentrations were chosen because it allowed us to meaningfully differentiate between additivity, synergy and antagonism (see below for details of classification method used). Briefly, drugs at high concentrations can kill off bacteria or render the population nearly dead. This makes it very difficult to distinguish between synergy and additivity, since additive expectations would be close to zero percent growth in cases where two single drugs by themselves each have a large effect on growth rate. Conversely, drugs at very-low concentrations have almost no effect on bacterial growth rate, making it difficult to distinguish between additivity and antagonism. In a section below, we formally define how the interaction types are classified.

\section{Drug interaction assay}

Experimental cell cultures were prepared with the method described above (see Growth conditions section), using $2 \mathrm{ml}$ of LB media supplemented with no drugs, each drug individually, and both drugs together at sub-ICs. For each experiment with a given drug pair, several concentrations resulting in subinhibitory (50-90\%) growth were used. For example, when testing the interaction between AZM and CPR, we used AZM 0.025, 0.03 and $0.035 \mu \mathrm{g} \mathrm{ml}^{-1}$ and CPR $0.01,0.015$ and $0.02 \mu \mathrm{g} \mathrm{ml}^{-1}$, resulting in nine different combinations for each individual experiment performed on this pair (see Table 1 for a full list of concentrations used). Each experiment on any given day included at least four duplicates of each drug concentration and each experiment was also repeated several times on multiple days to ensure validity. The number of successful trials per drug combination (excluding unsuccessful experiments or experiments with significant outliers) is listed here. TRI+FUS was successfully repeated five times, TRI+CLI five times, TRI+CHL four times, TRI+CPR seven times, TRI+VAN four times, TRI+NIT three times, TRI+TMP eight times, TRI+AMP four times, TRI+GEN four times, AZM+FUS three times, AZM+CLI twice, AZM+CHL three times, AZM+CPR twice, AZM+VAN seven times, AZM+NIT twice, AZM+TMP three times, AZM+AMP twice, $\mathrm{AZM}+\mathrm{GEN}$ four times, AZM+TRI five times, RIF+FUS nine times, RIF+CLI four times, RIF+CHL four times, RIF+CPR eight times, RIF+VAN twice, RIF + NIT three times, RIF+TMP five times, RIF+AMP four times, RIF+GEN twice, RIF+TRI seven times and RIF+AZM twice.

Table 1 Names, abbreviations, MICs and range of concentrations used for each antibiotic

\begin{tabular}{|c|c|c|c|c|}
\hline Antibiotic & Abbreviation & Concentration range $\left(\mu \mathrm{g} \mathrm{ml}^{-1}\right)$ & $M I C\left(\mu g I^{-1}\right)$ & Primary target \\
\hline Triclosan & TRI & $0.03-0.055$ & 0.060 & Fatty acid synthesis \\
\hline Rifampicin & RIF & $2-5$ & 16 & RNA polymerase \\
\hline Aztreonam & AZM & $0.025-0.045$ & 0.10 & Cell wall synthesis \\
\hline Ampicillin & AMP & $1-3$ & 6 & Cell wall synthesis \\
\hline Chloramphenicol & $\mathrm{CHL}$ & 1.5 & 8 & Protein synthesis, 50 S ribosome \\
\hline Ciprofloxacin & CPR & 0.015 & 0.02 & DNA gyrase \\
\hline Clindamycin & CLI & 75 & 225 & Protein synthesis, 50 S ribosome \\
\hline Fusidic Acid & FUS & $50-60$ & 300 & Protein synthesis, 50 S ribosome \\
\hline Gentamicin & GEN & $0.6-0.8$ & 0.8 & Protein synthesis, 30 S ribosome \\
\hline Nitrofurantoin & NIT & $2.5-3.5$ & 10 & DNA \\
\hline Trimethoprim & TMP & $0.15-0.18$ & 60 & Folic acid synthesis \\
\hline Vancomycin & VAN & 100 & 500 & Cell wall synthesis \\
\hline
\end{tabular}




\section{Classification of drug interactions}

Here, we use the same classification methods detailed in Segre et al. ${ }^{21}$ and Yeh et al. ${ }^{6}$ Additivity is defined as $W_{\mathrm{xy}}=W_{\mathrm{x}} W_{\mathrm{y}}$ where $W_{\mathrm{x}}$ is the proportion of growth versus the control with no drug; $W_{\mathrm{y}}$ is the proportion of growth with drug $\mathrm{Y}$; and $W_{\mathrm{xy}}$ is the proportion of growth with the drugs combined. For example, if drug $\mathrm{X}$ has a relative growth ratio of 0.6 (or 60\%) compared with the control with no drug, and drug $\mathrm{Y}$ has a relative growth ratio of 0.7 (or 70\%), the additive expectation of the two drugs together would be 0.42 (or $42 \%$ ). There is a range around 0.42 that would still be considered additive, for example, 0.43 . Formally, deviation from additivity is defined by $\tilde{\varepsilon}$, which is calculated from the formulas below. When $\tilde{\varepsilon}$ falls within the range of -1 to -0.5 , the interaction is classified as synergistic; when $\tilde{\varepsilon}$ is between -0.5 and 0.5 , the interaction is classified as additive; when $\tilde{\varepsilon}$ falls between 0.5 and 2 , the interaction is classified as antagonistic.

$$
\tilde{\varepsilon}=W_{\mathrm{xy}}-W_{\mathrm{x}} W_{\mathrm{y}} /\left|\tilde{W}_{\mathrm{xy}}-W_{\mathrm{x}} W_{\mathrm{y}}\right|
$$

$\tilde{W}_{\mathrm{xy}}=\min \left[W_{\mathrm{x}}, W_{\mathrm{y}}\right]$ for $W_{\mathrm{xy}}>W_{\mathrm{x}} W_{\mathrm{y}}$, and is 0 otherwise

If $\mathrm{W}_{\mathrm{xy}}>\min \left[W_{\mathrm{x}}, W_{\mathrm{y}}\right]$, then

$$
\tilde{\varepsilon}=\left[\left(W_{\mathrm{xy}}-\min \left[W_{\mathrm{x}}, W_{\mathrm{y}}\right]\right) /\left(1-\min \left[W_{\mathrm{x}}, W_{\mathrm{y}}\right]\right)\right]+1
$$

A pair was labeled inconclusive if the results from multiple experiments were inconsistent and thus could not be placed into a single category, as in Yeh et al. ${ }^{6}$ For example, of the eight experiments performed for TRI+TMP, the pair appears to demonstrate dose-specific interactions, with some interactions synergistic and others antagonistic. When the growth percentage for TMP treatment alone was $50 \%$ or less, the resulting pair was either additive or strongly synergistic. When the growth percentage for TMP alone was greater than 50\%, the resulting pair was either additive or antagonistic. Therefore, the pair as a whole could not be definitively categorized, since there were dose-dependent effects on the interaction type. TRI+RIF demonstrated $\tilde{\varepsilon}$ values that ranged from antagonistic suppression to synergy over seven duplicate experiments. Although there was a higher incidence of antagonism than synergy (5:2), no definitive conclusions can be made regarding the interaction between the pair. Similarly, TRI+AZM is inconclusive because its $\tilde{\varepsilon}$ values from five separate experiments range from additive to antagonistic suppression. Though a majority of experiments yielded an antagonistic result (3:2), it is not a large enough majority to categorize the pair as a whole is antagonistic.

\section{RESULTS}

Classification of pairwise interactions

Each of our thirty pair wise interactions was classified as additive, antagonistic or synergistic using $\tilde{\varepsilon}$ values as a measure of deviation from additivity (see Materials and methods section). Figure 2 displays the results in the format employed by Yeh et al. ${ }^{6}$ Percent residual growth versus growth in no drug LB medium is plotted for each single antibiotic and for the pair of antibiotics. Each bar graph represents one experiment performed on 1 day. Each pairwise combination experiment was repeated an average of four times, with each experiment having at least four determinations. The background color of each graph designates the form of epistasis where red is strong synergy $\left(\tilde{\varepsilon}_{\max }<-0.5\right)$; pink is weak synergy $\left(-0.5<\tilde{\varepsilon}_{\max }<-0.25\right)$; white is additive $\left(-0.25<\varepsilon_{\max }<0.5\right.$ and $\left.-0.5<\varepsilon_{\min }<0.25\right)$; green is antagonistic buffering $\left(0.25<\tilde{\varepsilon}_{\min }<1.15\right)$; blue is antagonistic suppression $\left(\tilde{\varepsilon}_{\min }>1.15\right)$; and gray is an inconclusive result. We have included representative epsilons for each drug pair in Table 2 to give the quantitative basis behind the categorization. These epsilon values correlate to the graphs shown in Figure 2.

Table 2 categorizes each pair by the type of interaction. The strongest and most notable of these synergies are shown in bold. TRI +TMP, TRI+RIF and TRI+AZM were inconclusive because their interactions did not fit the parameters for any single interaction category, based on the $\tilde{\varepsilon}$ values collected from multiple experiments (see Materials and methods section).

\section{Clustering of mechanistic groups}

In our previous works, we grouped 22 antibiotics into nine functional classes based on their interaction profiles with the other drugs. ${ }^{6,16}$ In this study, after testing RIF, TRI and AZM against one representative antibiotic from each of the nine functional classes, we analyzed their interaction profiles and incorporated them into the existing network (Figure 3). In Figure 3, dotted lines represent data from our previous work while solid lines represent data collected in this study. The red lines correspond only to strong synergies $\left(\tilde{\varepsilon}_{\max }<-0.5\right)$ but the green lines include both suppressive $\left(\tilde{\varepsilon}_{\min }>1.15\right)$ and buffering interactions $\left(0.25<\tilde{\varepsilon}_{\min }<1.15\right)$. Additive interactions $\left(-0.25<\tilde{\varepsilon}_{\max }<0.5\right.$ and $\left.-0.5<\tilde{\varepsilon}_{\min }<0.25\right)$ are not included in this figure, as they represent no interaction between antibiotics.

Contrary to our expectations, AZM did not group with the other cell wall synthesis inhibitors, represented by AMP, due to its interaction with RIF. Although AMP and RIF yield a synergistic interaction, AZM and RIF display a buffering interaction. This single conflict forced AZM to group independently.

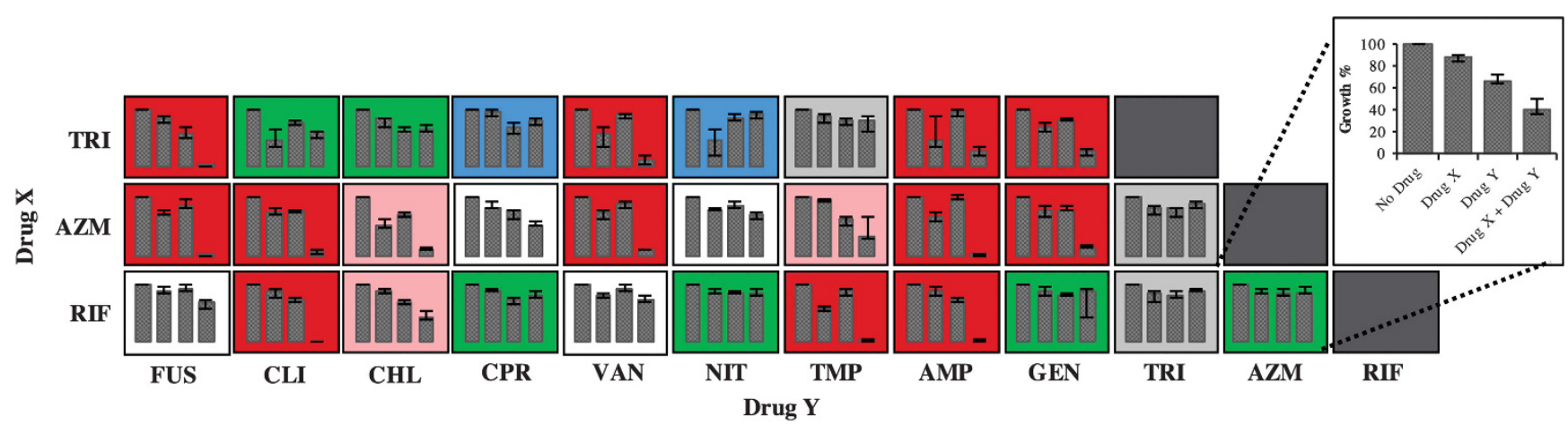

Figure 2 Systematic measurements of pairwise interactions between all combinations of antibiotics $\mathrm{X}$ and $\mathrm{Y}$. Each bar graph represents one experiment performed on one day for that drug pair. Within each panel, the bars, left to right, represent median growth rates of four replicates for cultures with: no drugs, drug $X$ only, drug $Y$ only, and the combination of the two drugs $X$ and $Y$. Growth rates are represented as percentages of the no drug control. Error bars represent the range of replicate measurements for each experiment. The background color of each graph designates the form of epistasis where red is strong synergy $\left(\tilde{\varepsilon}_{\max }<-0.5\right)$; pink is weak synergy $\left(-0.5<\tilde{\varepsilon}_{\max }<-0.25\right)$; white is additive $\left(-0.25<\tilde{\varepsilon}_{\text {max }}<0.5\right.$ and $\left.-0.5<\tilde{\varepsilon}_{\text {min }}<0.25\right)$; green is strong antagonistic buffering $\left(0.5<\tilde{\varepsilon}_{\min }<1.15\right)$; blue is antagonistic suppression $\left(\tilde{\varepsilon}_{\min }>1.15\right)$; and gray is an inconclusive result. See Table 1 for list of antibiotics used, their abbreviations, MIC's, and the range of concentrations tested for each antibiotic. 
Table 2 Categorization of pairwise interactions

\begin{tabular}{llll}
\hline Strong Synergy & & Weak Synergy & Additivity \\
\hline FUS+TRI (-0.98)a,b & RIF+TMP (-0.97) & AZM+CHL (-0.73) & RIF+FUS (-0.14) \\
AZM+FUS (-0.99) & RIF+AMP (-1.00) & RIF+CHL (-0.32) & AZM+CPR (-0.20) \\
AZM+CLI (-0.91) & RIF+CLI (-1.00) & AZM+TMP (-0.43) & AZM+NIT (0.28) \\
TRI+VAN (-0.73) & AZM+AMP (-0.99) & \\
TRI+AMP (-0.81) & AZM+VAN (-0.87) & \\
TRI+GEN (-0.52) & AZM+GEN (-0.75) & Antagonistic Suppression & Inconclusive \\
\hline Antagonistic Buffering & & TRI+CPR (1.35) \\
TRI+CLI (1.26) & RIF+GEN (1.21) & TRI+NIT (1.81) \\
TRI+CHL (1.09) & RIF+NIT (0.92) & TRI+TMP (0.80) \\
RIF+CPR (1.31) & AZM+RIF (0.99) & TRI+RIF (1.47) \\
\hline
\end{tabular}

Abbreviations: AMP, ampicillin; AZM, aztreonam; CHL, chloramphenicol; CLI, clindamycin; CPR, ciprofloxacin; FUS, fusidic acid; GEN, gentamicin; NIT, nitrofurantoin; RIF, rifampicin; TMP, trimethoprim; TRI, triclosan; VAN, vancomycin.

${ }^{a}$ Each pair is listed with its representative $\tilde{\varepsilon}$ value in parentheses. The $\tilde{\varepsilon}$ value of each pair listed here corresponds to the graphs shown in Figure 2 . Note that this table includes only one of many $\tilde{\varepsilon}$ values obtained for each pair, while the ultimate categorization of each pair was based on the overall range of $\tilde{\varepsilon}$ values obtained. Also note that for inconclusive pairs, the graphs shown in Figure 2 and therefore the $\tilde{\varepsilon}$ values given here do not reflect the wide range of results obtained which led to an inconclusive categorization.

${ }^{b}$ In this table, the strongest and most notable synergies, based on $\tilde{\varepsilon}$ values, are in bold.

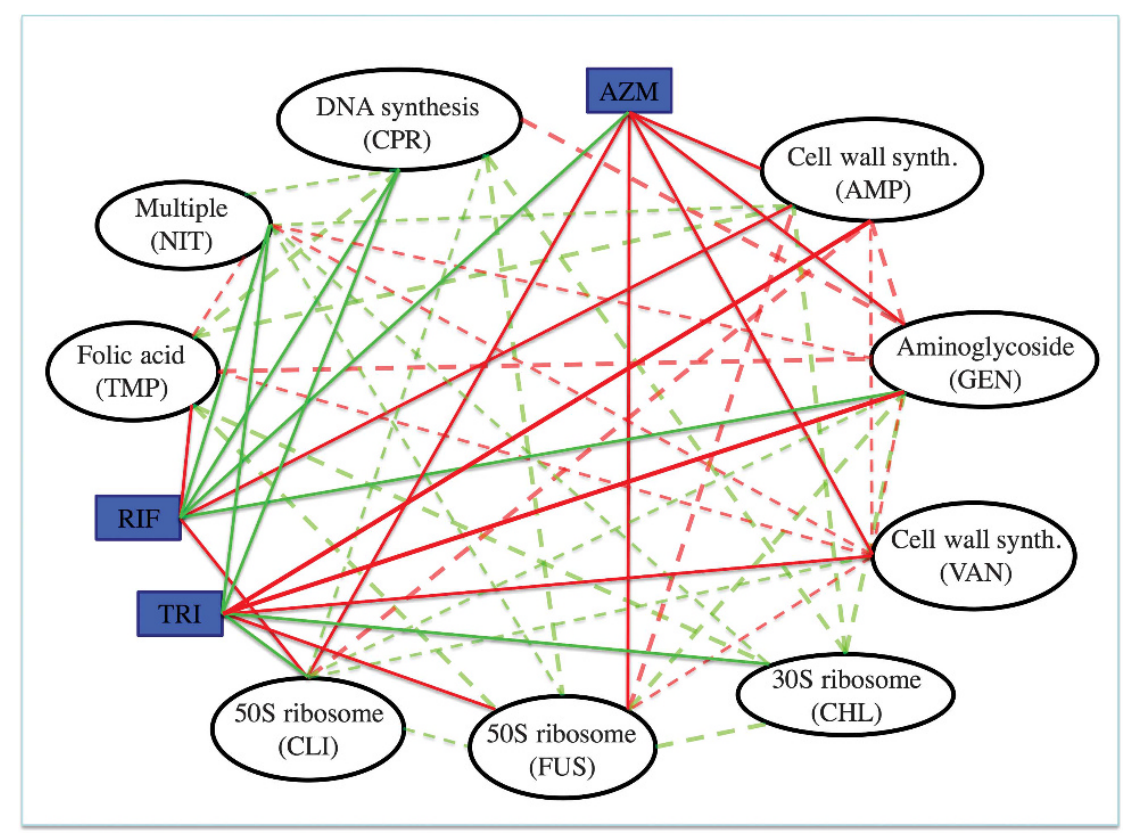

Figure 3 Unsupervised classification of the antibiotic network into monochromatically interacting classes of drugs into mechanisms of action. Red lines represent strong synergistic interactions between groups $\left(\tilde{\varepsilon}_{\max }<-0.5\right)$ green lines represent both antagonistic buffering and antagonistic suppression $\left(\tilde{\varepsilon}_{\max }>0.5\right)$. The dotted lines represent interactions that were found in our lab's previous work, 6,16 while solid lines represent interactions found in this work. In this representation, the interaction between vancomycin (VAN) and ampicillin (AMP) was changed to synergy after testing of the pair in wild-type E. coli, in contrast to the antagonistic interaction displayed in the mutant surA-strain. Rifampicin (RIF), aztreonam (AZM) and triclosan (TRI) are highlighted in blue to indicate that these drugs are the focus of this study. See Table 1 for list of antibiotics used, their abbreviations, and the range of concentrations tested for each antibiotic.

TRI, the fatty acid synthesis inhibitor, almost grouped into either the AZM or AMP group, except for a single conflict. TRI is synergistic with both AMP and AZM, which is a characteristic of drugs in the same functional class. However, it displays a conflicting interaction with CLI. Although AMP and AZM are both synergistic with CLI, TRI and CLI are antagonistic.

Finally, RIF, an RNA polymerase inhibitor, is synergistic with CLI and would have grouped with it in the $50 \mathrm{~S}$ ribosomal inhibitors, if not for its interaction with AZM. Although CLI and AZM are synergistic, RIF and AZM display antagonistic buffering. This single conflict forced RIF to group independently.
Based on their interaction profiles, RIF, TRI and AZM cannot be placed in any of the previously defined functional classes, while still keeping the interactions between classes monochromatic. Therefore, all three are placed into new functional classes of their own.

\section{DISCUSSION}

Our focus here is on increasing basic knowledge of drug-drug interactions in the context of a drug interaction network. Yeh et al. ${ }^{6}$ investigated pairwise interactions of 21 antibiotics in E. coli and found that the drug interaction network can be separated into classes of drugs that interact with each other either purely synergistically or 
purely antagonistically. Moreover, the classes correspond directly to the cellular functions affected by the antibiotics. We recently added VAN to this interaction network and found, surprisingly, that it defines a new functional class that is apart from other cell walltargeting drugs, such as AMP. ${ }^{16}$ This grouping is based on strong differences in their interactions with TMP and NIT (see Figure 3). Here, we have extended the interaction network by examining pairwise combinations involving RIF, TRI and AZM. These are widely used antibiotics, and given their initial targets, one would expect that at least RIF (RNA polymerase) and TRI (fatty acid biosynthesis) would define new functional classes. This is borne out by the resulting classifications (Figure 3) that show each of the three antibiotics to be in unique classes. AZM falls into a different class then either AMP or VAN, highlighting the complexity of drug-target interactions.

What is especially noteworthy is that, thus far, the addition of four drugs (including VAN from a previous study) has resulted in four new clustering groups. In particular, three different drugs that inhibit cell wall synthesis-AMP, VAN and AZM-all cluster into three distinct groups. We can speculate that aspects of their mechanisms of action may be sufficiently different, emanating either from their detailed interference with cell wall synthesis ${ }^{22-24}$ or from a pathway leading to hydroxl radical production and subsequent DNA damage. ${ }^{25}$ This can result in significant differences in their interaction with other drugs and thus in how they operate within a bacterial cell. This finding highlights the care that must be taken in choosing drugs for treatment because it is clear that not all drugs with superficially similar mechanisms of action ('cell wall synthesis') are similar with regards to their interactions with other drugs. Indeed, this result suggests that closer examination of mechanisms of action could reveal intriguing details into which drugs would best be used in which combinations. For example, AMP and VAN are both cell wall synthesis inhibitors. However, AMP interacts with TMP and NIT antagonistically, while VAN interacts with those same two drugs synergistically. Thus if one is looking for a synergistic interaction between two drugs based on mechanism of action, our results indicate that we cannot generalize and say that all cell wall synthesis inhibitors will be synergistic with all folic acid biosynthesis inhibitors.

We detected strong synergies between a number of drug pairs (Figure 2 and Table 2). In addition, pairs displaying antagonistic effects (colored green and blue in Figure 2; see also Materials and methods section) are of interest due to intriguing data from other studies that suggest antagonistic drug combinations could be used to combat the evolution of antibiotic resistance. ${ }^{26-29}$ The literature on synergistic and antagonistic combinations involving AZM, RIF and TRI is somewhat limited and involves different bacteria than what we use here. We have summarized the information from other studies in Table 3, where we have compared previous results with the data reported here. Of our 12 reported synergies, two have been found previously: AZM+VAN in E. coli $^{30}$ and AZM+GEN in Pseudomonas aeruginosa. ${ }^{15,31}$ In addition, the synergies between RIF+VAN and RIF +FUS have been suggested in several studies of Staphylococcus species, ${ }^{10,32}$ although the results were inconclusive and in both cases, further studies are warranted.

Several of our results also show conflicts with previous studies done in other bacteria. Where we found an antagonistic result for AZM + RIF, Nagaoka et al. ${ }^{33}$ found that $77.5 \%$ of P. aeruginosa strains used in their study showed additivity for this pair, while 5\% showed synergy. For RIF+CPR, we found an antagonistic interaction, whereas

Table 3 Comparison of previous literature findings

\begin{tabular}{|c|c|c|c|c|}
\hline Pair & Paper & Organism & Their findings & Our findings \\
\hline$A Z M+V A N$ & LaPlante 30 & E. coli, (Gram-negative) & $\begin{array}{l}\text { Synergy; defined as } \geqslant 2-\log 10 \text { decrease in CFU per } \mathrm{ml} \text { between pair } \\
\text { and lowest single drug }\end{array}$ & Strong synergy \\
\hline \multirow[t]{2}{*}{$A Z M+G E N$} & Araoka ${ }^{15}$ & P. aeruginosa (Gram-negative) & Synergy; defined as a decrease in the MIC of AZM & Strong synergy \\
\hline & Giamarellou $^{31}$ & P. aeruginosa (Gram-negative) & Synergistic in $29 / 30$ strains & \\
\hline \multirow[t]{7}{*}{$\mathrm{AZM}+\mathrm{CPR}$} & Chow $^{36}$ & P. maltophilia (Gram-negative) & Of 9 strains: & Additivity \\
\hline & & & 6 synergies, defined as sum FIC of both drugs $\leqslant 0.5$ & \\
\hline & & & $\begin{array}{l}2 \text { additive, } 1 \text { indifferent, both defined as sum FIC between } 0.5 \text { and } 4 \text {. } \\
\text { (FIC = fractional inhibitory concentration) }\end{array}$ & \\
\hline & Sader ${ }^{37}$ & P. aeruginosa, Enterobacteria & Of 40 strains each: & \\
\hline & & (Gram-negative) & P. aeruginosa: $12.5 \%$ synergy, $45 \%$ partial synergy, $37.5 \%$ additivity. & \\
\hline & & & Enterobacteria: $2.5 \%$ synergy, $52 \%$ partial synergy, $17.5 \%$ additivity. & \\
\hline & & & Interactions defined by change in MIC. & \\
\hline \multirow[t]{3}{*}{$\mathrm{AZM}+\mathrm{RIF}$} & Nagaoka ${ }^{33}$ & P. aeruginosa (Gram-negative) & Of 40 strains: & Antagonism \\
\hline & & & $5 \%$ synergy, $77.5 \%$ additive & \\
\hline & & & Interactions defined by FIC. & \\
\hline \multirow[t]{2}{*}{$\mathrm{RIF}+\mathrm{VAN}$} & Gagnon 10 & S. epidermidis (Gram-positive) & Slow synergy & Weak synergy \\
\hline & Russell ${ }^{32}$ & S. aureus (Gram-positive) & $\begin{array}{l}\text { Review of previous studies found conflicting results. either improves } \\
\text { health outcomes or has no effect on patients with } S \text {. aureus. }\end{array}$ & \\
\hline $\mathrm{RIF}+\mathrm{GEN}$ & Gagnon 10 & S. epidermidis (Gram-positive) & $\begin{array}{l}\text { Antagonism. (With other aminoglycosides amikacillin and tobramycin, } \\
\text { RIF displayed synergy and additivity respectively.) }\end{array}$ & Antagonism \\
\hline \multirow[t]{3}{*}{$\mathrm{RIF}+\mathrm{CPR}$} & Gagnon ${ }^{10}$ & S. epidermidis (Gram-positive) & Slow synergy & Antagonism \\
\hline & Boisivon 46 & L. monocytogenes & Antagonism. & \\
\hline & & (Gram-positive) & Prevented mutants when used at greater than MIC of both drugs. & \\
\hline \multirow[t]{2}{*}{$\mathrm{RIF}+\mathrm{FUS}$} & Farber $^{47}$ & Staphylococci (Gram-positive) & Of 20 strains: & Weak synergy \\
\hline & & & 3 synergy, 17 additivity. & \\
\hline
\end{tabular}

Abbreviations: AMP, ampicillin; AZM, aztreonam; CHL, chloramphenicol; CLI, clindamycin; CPR, ciprofloxacin; FUS, fusidic acid; GEN, gentamicin; NIT, nitrofurantoin; RIF, rifampicin; TMP, trimethoprim; TRI, triclosan; VAN, vancomycin. 
Gagnon et al. ${ }^{10}$ found a slow synergy in S. epidermidis, a Gram-positive bacteria. These authors also performed antibiotic interaction assays with RIF and the cell wall synthesis inhibitors cloxacillin, cephalothin, cefazolin and cefamandole and found that RIF with these cell wall inhibitors displayed rapid synergy. ${ }^{10}$ In contrast, we found a wide array of interactions between RIF and other cell wall inhibitors in E. coli. In our experiments, we see that RIF+AMP is synergistic, RIF +VAN is additive and RIF+AZM is antagonistic. These differing interactions with RIF helped in grouping these cell wall synthesis inhibitors independently. It has also been reported that in humans, RIF increases the metabolism of CHL and CLI, lowering their serum concentration. $^{34,35}$ Therefore, although we found weak synergies between RIF and these two drugs, clinical use may be challenging. Finally, using Fractional Inhibitory Concentration Indices, Chow et al. ${ }^{36}$ and Sader et al. ${ }^{37}$ report several instances of synergy when testing $\mathrm{AZM}+\mathrm{CPR}$ in multiple strains of $P$. maltophilia and $P$. aeruginosa, respectively, which we did not detect in E. coli. Although we use a slightly different method for determining synergy and antagonism, our method is very conservative, meaning that an interaction would have to be strongly synergistic for us to term it synergistic. ${ }^{6}$ Thus the more parsimonious explanation for these differences is that that the interactions between drugs may be bacteria dependent, rather than universal. Although this is not surprising, a thorough understanding of how drug-drug interactions differ across a range of bacterial species is lacking in the literature.

The focus of many labs and pharmaceutical companies has been on finding drugs with novel mechanisms of action. We describe here three well-known drugs that cluster into new and distinct groups compared with the original study (Yeh et al. ${ }^{6}$ ) that first described how to cluster drugs based on mechanisms of action. This suggests several things. First, any new group is of potential interest because it denotes a different mechanism of action, or some difference significant enough to be clustered in a separate group. Second, it would be worthwhile to expand the study of drug-drug interactions into more drugs as well as potentially more bacteria (for example, gram-positive bacteria) to examine drug interactions and the resulting clustering. (A systematic study of the interaction network of antifungal drugs in Saccharomyces cerevisiae has been carried out). ${ }^{38-40}$ Third, a commonly used and potentially overused antimicrobial, TRI, clusters in its own group, indicating that the mechanism of action of TRI is quite different from all 24 other drugs tested here and previously. Although TRI is a topical agent, and there may be limits of this study to practical application, results from this work suggests that TRI could have unique drug properties and thus should be more judiciously used. In addition, recent research indicates that small amounts of chemicals considered safe, including TRI, might actually be carcinogenic in combination with other chemicals considered safe. ${ }^{41}$

We note that there are several limitations to our study. The first is that we focus on interactions as measured by single concentrations of each drug, called Bliss Independence. ${ }^{42}$ This is a more simple and straightforward method for examining interactions, as compared with measuring interactions over an entire range of drug concentrations per drug, 'the checkerboard method'. ${ }^{43-45}$ We use Bliss Independence as a straightforward method of testing for interactions at specific concentrations, and we acknowledge that within two-drug combinations, the interaction could vary somewhat based on concentrations chosen. Loewe ${ }^{43}$ identified this issue and indeed, a full-range of drug concentrations may yield a fuller picture of interactions. However, we expect that different drug concentrations typically do not change an interaction from synergy to antagonism or vice versa, though we do see interactions change from additivity to synergy or antagonism, and vice versa. ${ }^{6,16}$ Furthermore, we sought to keep our experimental methods as closely aligned with those conducted previously on drug interaction networks, ${ }^{6}$ as our research is a natural extension of that work.

Second, we also limit our study to sub-ICs of drugs in LB media and the interactions that result from slowing growth rate, rather than examining higher concentrations in other media such as Mueller Hinton Broth and their interactions for killing bacteria. As this study is expanding on a conceptual framework for drug interactions, we decided to use these lower concentrations to examine interactions in keeping with the original study. ${ }^{6}$ Variations on these parameters of the study could be an interesting and useful follow up to this work.

In summary, we find here that we can expand the clustering of drugs to more distinct groups than proposed in previous studies. ${ }^{6,16}$ Specifically, we find that the antimicrobials TRI, RIF and AZM all cluster in their own groups, indicating significantly different mechanisms of action. Some drugs with similar mechanisms of action (for example, cell wall synthesis inhibitors AZM, VAN and AMP) may not cluster with each other, as initially expected. This indicates that there may be important differences in the details of these antibiotics' mechanisms of action, even if they all broadly affect cell wall synthesis. Drug-drug interaction networks can be used to reveal which drugs exhibit such differences and therefore help to optimize the use of combinatorial drug therapies in clinical contexts. We also found several new synergistic combinations that could be useful clinically. We suggest that examining drug-drug interactions in a network context could prove useful both clinically and for basic understanding of drug mechanisms of action.

\section{CONFLICT OF INTEREST}

The authors declare no conflict of interest.

\section{ACKNOWLEDGEMENTS}

PY was supported by the Hellman Fellows Fund and a UCLA Faculty Career Development Award. We thank Elinne Becket for her assistance with the figures.

1 Alekshun, M. N. \& Levy, S. B. Molecular mechanisms of antibacterial multidrug resistance. Cell 128, 1037-1050 (2007).

2 Davies, J. Microbes have the last word. EMBO Rep. 8, 616-621 (2007).

3 Nikaido, H. Outermembrane barrier as a mechanism of antimicrobial resistance. Antimicrob. Agents Chemother. 33, 1831-1836 (1989).

4 Cottarel, G. \& Wierzbowski, J. Combination drugs, an emerging option for antibacterial therapy. Trends Biotechnol. 25, 547-555 (2007).

5 Bollenbach, T. Antimicrobial interactions: mechanisms and implications for drug discovery and resistance evolution. Curr. Opin. Microbiol. 27, 1-9 (2015).

6 Yeh, P., Tschumi, A. I. \& Kishony, R. Functional classification of drugs by properties of their pairwise interactions. Nat. Genet. 38, 489-494 (2006).

7 Campbell, E. A. et al. Structural mechanism for rifampicin inhibition of bacterial RNA polymerase. Cell 104, 901-912 (2001).

8 Musser, J. M. Antimicrobial agent resistance in mycobacteria: molecular genetic insights. Clin. Microbiol. Rev. 8, 496-514 (1995).

9 Aboltins, C. A. et al. Treatment of staphylococcal prosthetic joint infections with debridement, prosthesis retention and oral rifampicin and fusidic acid. Clin. Microbiol. Infect. 13, 586-591 (2007).

10 Gagnon, R. F., Richards, G. K. \& Kostiner, G. B. Time-kill efficacy of antibiotics in combination with rifampicin against Staphylococcus epidermidis biofilms. ADV Perit. Dial. 10, 189-192 (1994).

11 Russell, A. D. Whither triclosan? J. Antimicrob. Chemother. 53, 693-695 (2004).

12 Levy, S. B. Antibacterial household products: cause for concern. Emerg. Infect. Dis 7, 512-515 (2001).

13 McMurry, L. M., Oethinger, M. \& Levy, S. B. Triclosan targets lipid synthesis. Nature 394, 531-532 (1998).

14 Terico, A. T. \& Gallagher, J. C. Beta-lactam hypersensitivity and cross-reactivity. J. Pharm. Pract. 27, 530-544 (2014). 
15 Araoka, H. et al. In vitro combination effects of aztreonam and aminoglycosides against multidrug-resistant Pseudomonas aeruginosa in Japan. Jpn. J. Infect. Dis. 65 84-87 (2012)

16 Zhou, A. et al. Synergistic interactions of vancomycin with different antibiotics against Escherichia coli: trimethoprim and nitrofurantoin display strong synergies with vancomycin against wild-type E. coli. Antimicrob. Agents Chemother. 59, 276-281 (2015)

$17 \mathrm{Baba}, \mathrm{T}$. et al. Construction of Escherichia coli K-12 in-frame, single-gene knockout mutants: the Keio collection. Mol. Syst. Biol. 2, 20060008 (2006).

18 Datsenko, K. A. \& Wanner, B. L. One-step inactivation of chromosomal genes in Escherichia coli K-12 using PCR products. Proc. Natl Acad. Sci. USA 97 6640-6645 (2000).

19 Miller, J. H. A Short Course in Bacterial Genetics: A Laboratory Manual and Handbook for Escherichia coli and Related Bacteria, (Cold Spring Harbor Laboratory Press, Cold Spring Harbor, NY, USA, 1992).

20 Andrews, J. M. Determination of minimum inhibitory concentrations. J. Antimicrob. Chemother. 48( Suppl 1), 5-16 (2001).

21 Segre, D., Deluna, A., Church, G. M. \& Kishony, R. Modular epistasis in yeast metabolism. Nat. Genet. 37, 77-83 (2005).

22 Waxman, D. J. \& Strominger, J. L. Penicillin-binding proteins and the mechanism of action of beta-lactam antibiotics. Annu. Rev. Biochem. 52, 825-869 (1983).

23 Hammes, W. P. \& Neuhaus, F. C. On the mechanism of action of vancomycin: inhibition of peptidoglycan synthesis in Gaffkya homari. Antimicrob. Agents Chemother. 6, 722-728 (1974).

24 Georgopapadakou, N. H., Smith, S. A. \& Sykes, R. B. Mode of action of azthreonam. Antimicrob. Agents Chemother. 21, 950-956 (1982).

25 Kohanski, M. A., Dwyer, D. J., Hayete, B., Lawrence, C. A. \& Collins, J. J. A common mechanism of cellular death induced by bactericidal antibiotics. Cell 130, 797-810 (2007).

26 Yeh, P. J., Hegreness, M. J., Aiden, A. P. \& Kishony, R. Drug interactions and the evolution of antibiotic resistance. Nat. Rev. Microbiol. 7, 460-466 (2009)

27 Hegreness, M., Shoresh, N., Damian, D., Hartl, D. \& Kishony, R. Accelerated evolution of resistance in multidrug environments. Proc. Natl Acad. Sci. USA 105, 13977-13981 (2008).

28 Michel, J. B., Yeh, P. J., Chait, R., Moellering, R. C. Jr. \& Kishony, R. Drug interactions modulate the potential for evolution of resistance. Proc. Natl Acad. Sci. USA 105 14918-14923 (2008).

29 Chait, R., Craney, A. \& Kishony, R. Antibiotic interactions that select against resistance. Nature 446, 668-671 (2007).

30 LaPlante, K. L. \& Sakoulas, G. Evaluating aztreonam and ceftazidime pharmacodynamics with Escherichia coli in combination with daptomycin, linezolid, or vancomycin in an in vitro pharmacodynamic model. Antimicrob. Agents Chemother. 53, 4549-4555 (2009).

31 Giamarellou, H., Zissis, N. P., Tagari, G. \& Bouzos, J. In vitro synergistic activities of aminoglycosides and new beta-lactams against multiresistant Pseudomonas aeruginosa. Antimicrob. Agents Chemother. 25, 534-536 (1984).
32 Russell, C. D., Lawson McLean, A., Saunders, C. \& Laurenson, I. F. Adjunctive rifampicin may improve outcomes in Staphylococcus aureus bacteraemia: a systematic review. J Med Microbiol 63, 841-848 (2014).

33 Nagaoka, R. et al. [In vitro combined effects of double antibacterial drugs against multidrug-resistant Pseudomonas aeruginosa isolates: comparison among combinations of colistin, arbekacin, aztreonam, rifampicin and piperacillin]. Jpn. J. Antibiot. 67, 167-174 (2014).

34 Niemi, M., Backman, J. T., Fromm, M. F., Neuvonen, P. J. \& Kivisto, K. T. Pharmacokinetic interactions with rifampicin: clinical relevance. Clin. Pharmacokinet. 42, 819-850 (2003).

35 Bernard, A. et al. Dramatic reduction of clindamycin serum concentration in staphy lococcal osteoarticular infection patients treated with the oral clindamycin-rifampicin combination. J. Infect. 71, 200-206 (2015).

36 Chow, A. W., Wong, J. \& Bartlett, K. H. Synergistic interactions of ciprofloxacin and extended-spectrum beta-lactams or aminoglycosides against multiply drugresistant Pseudomonas maltophilia. Antimicrob. Agents Chemother. 32 , 782-784 (1988).

37 Sader, H. S., Huynh, H. K. \& Jones, R. N. Contemporary in vitro synergy rates for aztreonam combined with newer fluoroquinolones and beta-lactams tested against gram-negative bacilli. Diagn. Microbiol. Infect. Dis. 47, 547-550 (2003).

38 Cokol, M. et al. Large-scale identification and analysis of suppressive drug interactions. Chem. Biol. 21, 541-551 (2014).

39 de Vos, M. G. \& Bollenbach, T. Suppressive drug interactions between antifungals. Chem. Biol. 21, 439-440 (2014).

$40 \mathrm{Cokol}, \mathrm{M}$. et al. Systematic exploration of synergistic drug pairs. Mol. Syst. Biol. 7 , 544 (2011).

41 Goodson, W. H. 3rd et al. Assessing the carcinogenic potential of low-dose exposures to chemical mixtures in the environment: the challenge ahead. Carcinogenesis 36 (Suppl 1), S254-S296 (2015).

42 Bliss, C. I. The toxicity of poisons applied jointly. Ann. Appl. Biol. 26, 585-615 (1939).

43 Loewe, S. The problem of synergism and antagonism of combined drugs. Arzneimittelforschung 3, 285-290 (1953).

44 Elion, G. B., Singer, S., Hitchings, G. H., Balis, M. E. \& Drown, G. B. Effects of purine antagonists on a diaminopurine-resistant strain of Lactobacillus casei. J Biol Chem 202 647-654 (1953).

45 Odds, F. C. Synergy, antagonism, and what the chequerboard puts between them. J. Antimicrob. Chemother. 52, 1 (2003).

46 Boisivon, A., Guiomar, C. \& Carbon, C. In vitro bactericidal activity of amoxicillin, gentamicin, rifampicin, ciprofloxacin and trimethoprim-sulfamethoxazole alone or in combination against Listeria monocytogenes. Eur. J. Clin. Microbiol. Infect. Dis. 9 , 206-209 (1990).

47 Farber, B. F., Yee, Y. C. \& Karchmer, A. W. Interaction between rifampin and fusidic acid against methicillin-resistant coagulase-positive and -negative staphylococci. Antimicrob. Agents Chemother. 30, 174-175 (1986). 